\section{O sexo masculino vulnerável: razão de masculinidade entre os óbitos fetais brasileiros}

\author{
The vulnerable male, or the sex ratio among \\ fetal deaths in Brazil
}

Alexandre Dias Porto Chiavegatto Filho 1 Ruy Laurenti 1

\title{
Introdução
}

1 Faculdade de Saúde Pública, Universidade de São Paulo, São Paulo, Brasil.

Correspondência A. D. P. Chiavegatto Filho Faculdade de Saúde Pública, Universidade de São Paulo. Av. Dr. Arnaldo 715, São Paulo, SP 01246-904, Brasil. alexdiasporto@usp.br

\begin{abstract}
Some studies indicate the existence of innate
Abstract male vulnerabilities, especially during the perinatal period. The current study is a cross-sectional analysis of fetal mortality in Brazil according to sex from 2000 to 2009, stratified by maternal characteristics (age, schooling, and gestational age), using Ministry of Health data (DATASUS). The analysis included all fetal deaths from 2000 to 2009, except when the sex of the fetus was not recorded. The malelfemale sex ratio (SR) for all fetal deaths was 1.188. Analysis of maternal characteristics showed that the SR was statistically higher $(p<0.01)$ in mothers that were younger (10-14 years), had no formal schooling, and with gestational age $<22$ weeks. The study showed a statistically higher-than-expected $S R(p<0.01)$ for 13 underlying causes of death and a lower SR for two others. The results suggest a potential innate male vulnerability.
\end{abstract}

Sex Ratio; Fetal Mortality; Men's Health
Homens apresentam maior taxa de mortalidade que mulheres em praticamente todas as faixas etárias e para a maioria das doenças, o que resulta em uma menor expectativa de vida (aproximadamente de cinco anos em países desenvolvidos) 1 . Como justificativa para essa diferença, diversos estudos apontam a importância de alguns fatores de risco mais comuns entre homens, destacando-se maior presença de alcoolismo, tabagismo e ações violentas 2.

Por outro lado, alguns autores defendem a existência de diferenças biológicas inatas entre homens e mulheres, importantes para esse padrão de mortalidade. Segundo essa hipótese, homens seriam naturalmente mais vulneráveis ao efeito imediato de algumas doenças, o que ajudaria a explicar tanto a maior mortalidade, quanto a menor morbidade masculinas 3 .

A análise da importância desses dois fatores como explicativos da maior mortalidade entre homens é uma das grandes questões atuais dos estudos de gênero. Alguns autores defendem a análise da mortalidade infantil com o objetivo de excluir os fatores de risco mais presentes entre homens adultos, mas estudos apontam que influências externas podem também afetar a sobrevivência do indivíduo no primeiro ano de vida ${ }^{4}$. Pesquisas mostram que, em países em desenvolvimento, principalmente nas suas regiões mais pobres, crianças e recém-nascidos do sexo 
masculino podem ser priorizados no acesso a melhor alimentação e cuidados de saúde, por razões normalmente associadas a maiores expectativas de retornos financeiros no futuro 5 .

A maior parte dos estudos sobre a razão de masculinidade (RM) tem analisado a proporção de homens entre os nascidos vivos. Taxas maiores de recém-nascidos do sexo feminino já foram estatisticamente associadas a desastres naturais, maiores níveis de poluição, depressão econômica e até ao ataque terrorista de 11 de setembro de 2001 6. Em comum, esses eventos parecem indicar um possível efeito de situações de estresse no maior nascimento proporcional de mulheres 7 . O problema desse tipo de investigação é que, ao estudar somente os nascidos vivos segundo sexo, não é possível identificar as razões específicas que levaram a essa diferença, isto é, ao não incluírem uma análise dos óbitos fetais segundo causa básica, torna-se difícil identificar como esses fatores de risco têm levado ao nascimento de mais mulheres do que de homens. O uso de dados de nascidos vivos pela maioria dos estudos tem sido justificado pelo fato de que dados de mortalidade fetal muitas vezes não são confiáveis nem se encontram disponíveis com periodicidade na maior parte dos países ${ }^{8}$.

De acordo com uma revisão de Fonseca \& Coutinho ${ }^{9}$, a maior parte dos estudos brasileiros sobre mortalidade perinatal (referente a óbitos ocorridos entre a 22a semana de gravidez e o sétimo dia pós-parto) tem se concentrado na neomortalidade, sendo a análise específica das causas de óbito fetais ainda pouco utilizada. Uma recente avaliação de 10.072 puérperas, no Rio de Janeiro, verificou associação estatisticamente significativa entre idade materna e óbitos neonatais, mas não no que se refere a óbitos fetais 10 . Um estudo de uma coorte de nascimentos realizado em São Paulo encontrou associação estatisticamente significativa entre óbito fetal e baixa escolaridade da mãe (menor que quatro anos) 11 . Ao analisar mortalidade perinatal segundo sexo, uma pesquisa realizada em Pelotas (Rio Grande do Sul) verificou associação entre sexo masculino e maior mortalidade perinatal, mas não no que diz respeito a mortalidade fetal 12 .

Em uma revisão bibliográfica de óbitos perinatais, Lansky et al. 13 concluíram que, no Brasil, a maioria desses óbitos pode ser classificada como “evitável”. Ainda conforme os autores, o país tem atualmente taxas de mortalidade infantil semelhantes às que os países desenvolvidos tinham no final da década de 60 .

A análise da mortalidade fetal em separado da mortalidade perinatal torna-se necessária porque, enquanto tem ocorrido uma expressiva redução da neomortalidade no Brasil, a morta- lidade fetal permanece ainda bastante elevada 9 . Investigar a mortalidade fetal segundo sexo pode também ajudar na identificação da existência de vulnerabilidades biológicas masculinas e apontar possibilidades de prevenção.

\section{Metodologia}

Os dados utilizados na análise foram os óbitos fetais, disponibilizados pelo Sistema de Informações sobre Mortalidade (SIM) do Ministério da Saúde. Óbito fetal é definido como "a morte de um produto de concepção antes da expulsão do corpo da mãe, independente da duração da gravidez. A morte do feto é caracterizada pela inexistência, depois da separação, de qualquer sinal descrito para o nascido vivo" 14 (p. 29). Atualmente, no Brasil, é obrigatório o preenchimento de uma declaração de óbito fetal quando a gestação tiver duração igual ou superior a 20 semanas ou o feto tiver peso corporal igual ou superior a 500 gramas e/ou estatura igual ou superior a $25 \mathrm{~cm} 14$.

Foi realizado um estudo do tipo transversal, avaliando a diferença entre a mortalidade fetal masculina e feminina, com base no conceito de RM, em que o número de óbitos masculinos é dividido pelo número de óbitos femininos. Assim, no caso de serem verificados 105 óbitos masculinos e 100 femininos, a RM será 105/100 = 1,05; em outras palavras, ocorreram 105 óbitos masculinos para cada 100 femininos. De forma equivalente, a RM pode ser convertida em uma proporção; no exemplo anterior, 105/205 = 51,22\% dos óbitos foram do sexo masculino.

O período estudado foram os dez anos entre 2000 e 2009 (inclusive), sendo o último o ano mais recente disponível atualmente para análise. Os 352.992 óbitos fetais ocorridos no território brasileiro durante esse período foram incluídos na análise, excetuando-se os 13.109 que apresentaram sexo ignorado na declaração de óbitos $(13.109 / 352.992=3,7 \%)$.

As variáveis avaliadas foram selecionadas com o objetivo de representarem características da mãe e da gestação que possam ter influência no óbito: idade materna, escolaridade materna e duração da gravidez. Em relação a cada variável, foram utilizadas as categorias estabelecidas pelo Departamento de Informática do SUS (DATASUS; http://www.datasus.gov.br), com exceção da idade materna, sendo as categorias acima de 40 anos de idade agrupadas em uma só. Para o cálculo do p-valor, utilizou-se a análise de teste para proporção unilateral com distribuição normal padrão (Z). As causas básicas selecionadas para análise foram as mais frequentes entre os óbitos 
fetais, utilizando-se as denominações da Classificação Internacional de Doenças, 10a revisão (CID-10) 15. Para as análises estatísticas, foi utilizado o software Stata 11 (Stata Corp., College Station, Estados Unidos).

\section{Resultados}

A RM para o total de óbitos fetais analisados foi de 1,188 , indicando que, para cada mil óbitos femininos, ocorreram 1.188 óbitos masculinos. Esse valor é consideravelmente maior do que a RM para nascidos vivos, cujo valor de 1,05 é um dos mais consistentemente encontrados na área de saúde pública 16 .

A Figura 1 apresenta as diferentes razões de masculinidade para as três variáveis analisadas (idade da gestante, escolaridade da gestante e duração da gestação) e suas respectivas categorias. No caso da idade da gestante, a RM é maior para as idades mais extremas (de 1,34 entre gestantes com 10 a 14 anos e de 1,23 entre 40 e mais anos), atingindo o seu ponto mínimo para gestantes entre 20 a 24 anos $(1,16)$. Das três variáveis analisadas, a escolaridade da gestante é aquela que apresenta menor variabilidade de RM entre as categorias, de 1,17 para gestantes com um a três anos de escolaridade a 1,23 para aquelas com nenhuma escolaridade. Os valores mais extremos de RM foram verificados entre duas categorias da duração da gravidez: 1,48 para gestações com menos de 22 semanas e 1,13 para gestações entre 28 e 31 semanas. Todas as razões de masculinidade analisadas ficaram acima das verificadas entre nascidos vivos $(1,05)$.

A Tabela 1 apresenta uma análise detalhada dos valores de RM apresentados na Figura 1 e das diferenças estatisticamente significativas. Para a variável idade da gestante, a categoria mais frequente foi a de 20 a 24 anos (total de 72.539 óbitos fetais); a menos frequente foi a de 10 a 14 anos (3.254 óbitos). Esta categoria também apresentou RM mais elevada entre as analisadas. A RM de 1,339 (com população de 3.254) foi estatisticamente maior $(\mathrm{p}<0,05)$ do que o verificado em todas as outras categorias. O mesmo foi observado para a categoria de 20 a 24 anos, sendo neste caso menor que todas as outras $(1,158)$.

Para a variável escolaridade da gestante, a categoria "nenhuma escolaridade" apresentou a maior RM $(1,232)$ e foi a única com RM estatisticamente diferente de todas as outras. As outras analisadas só foram diferentes de, no máximo, duas outras (uma delas sendo a de "nenhuma escolaridade", em todos os casos). Para a variável duração da gestação, a maior RM verificada entre todas as categorias do estudo (1,479 verificada para gestações com menos de 22 semanas) foi também estatisticamente diferente das outras $(\mathrm{p}<0,05)$. As gestações de 28 a 31 semanas apresentaram RM estatisticamente menor $(1,134)$ em relação a todas as outras.

A Tabela 2 analisa as causas básicas mais frequentes para o total de óbitos fetais e para as categorias com maior RM entre as variáveis analisadas anteriormente. Incluindo todos os óbitos, as maiores razões de masculinidade encontradas foram, em ordem decrescente, para as seguintes causas básicas: as decorrentes de gestações de curta duração e baixo peso (RM de 1,50 ), transtornos transitórios do metabolismo de carboidratos $(1,38)$ e hidrocefalia congênita $(1,35)$. As menores razões foram verificadas nos casos de anencefalia $(0,59)$ e afecções que comprometem o tegumento $(0,95)$.

A análise da diferença estatisticamente significante foi realizada em relação à RM verificada para recém-nascidos $(1,05)$ no período de análise (2000 a 2009). As razões de masculinidade foram estatisticamente maiores para 13 causas básicas e estatisticamente menores para as duas causas mencionadas anteriormente.

$\mathrm{Na}$ análise das causas básicas segundo as categorias com maior RM de cada uma das três variáveis, verifica-se que algumas apresentam RM estatisticamente diferente de 1,05 em todas as categorias analisadas, sendo elas: complicações maternas da gravidez; complicações da placenta, cordão umbilical e membranas; hipóxia intrauterina; morte fetal não classificada em outra parte. No caso de RM menor que 1,05, a anencefalia foi estatisticamente significativa para todas as categorias, exceto para gestação com menos de 22 semanas.

\section{Discussão}

Os resultados encontrados pelo estudo estão de acordo com a teoria da vulnerabilidade biológica inata masculina. Entre as três variáveis analisadas (idade da gestante, escolaridade da gestante e período de gestação), as categorias mais relacionadas com maior risco (idade entre $10 \mathrm{e}$ 14 anos, nenhuma escolaridade e gestação com menos de 22 semanas) apresentaram maior RM, sendo esses valores, em todos os casos, estatisticamente maiores do que nas outras categorias analisadas $(\mathrm{p}<0,05)$.

Estudos apontam que situações de estresse levam a um aumento da RM fetal devido à maior vulnerabilidade dos fetos masculinos aos corticosteroides liberados em situações extremas 6 . A gravidez na infância ou adolescência no Brasil está associada a pressões e rejeições familiares, 
Figura 1

Razão de masculinidade (RM) dos óbitos fetais segundo idade da gestante, escolaridade da gestante e duração da gestação. Brasil, 2000 a 2009

1a) Idade da gestante

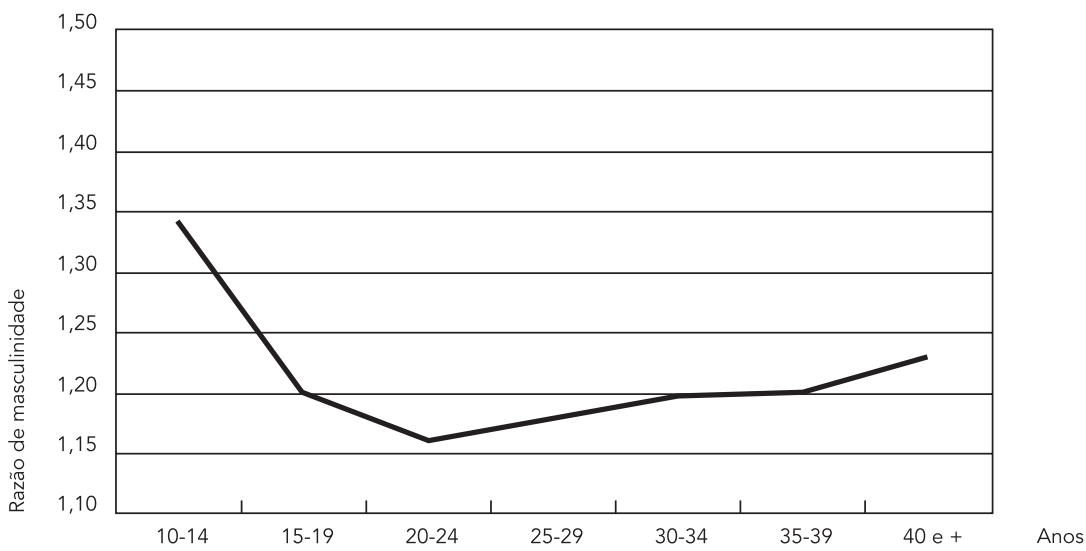

1b) Escolaridade da gestante

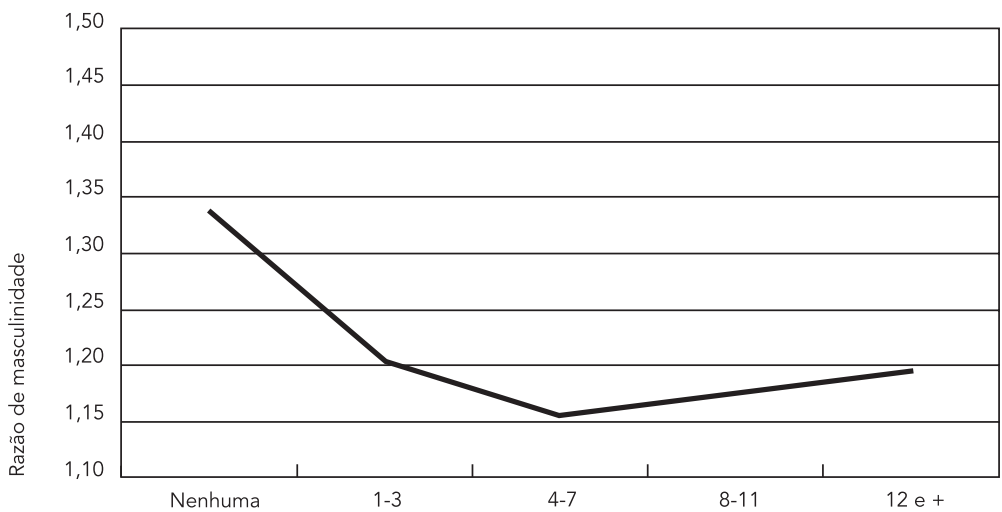

Escolaridade (anos)

1c) Duração da gestação

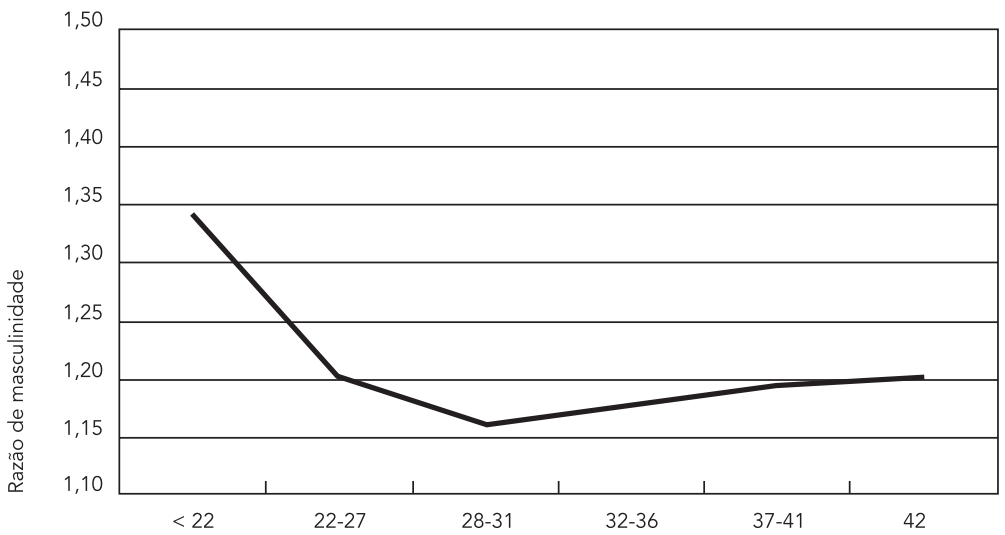

Semanas 
Óbitos fetais segundo as variáveis idade da gestante, escolaridade da gestante e duração da gestação. Brasil, 2000 a 2009.

\begin{tabular}{|c|c|c|c|c|}
\hline & $\begin{array}{c}\text { Masculino } \\
(\mathrm{N}=184.564)\end{array}$ & $\begin{array}{c}\text { Feminino } \\
(\mathrm{N}=155.319)\end{array}$ & $\begin{array}{c}\text { Razão de } \\
\text { masculinidade } \\
\text { (total }=1,188)\end{array}$ & $\begin{array}{c}\text { Valor de } \mathrm{p}<0,05 \\
\text { (em relação a) }\end{array}$ \\
\hline \multicolumn{5}{|c|}{ 1. Idade da gestante (anos) } \\
\hline 1a. $10-14$ & 1.863 & 1.391 & 1,339 & $\begin{array}{c}1 \mathrm{~b}, 1 \mathrm{c}, 1 \mathrm{~d}, 1 \mathrm{e} \\
1 \mathrm{f}, 1 \mathrm{~g}\end{array}$ \\
\hline 1b. $15-19$ & 29.139 & 24.239 & 1,202 & $1 \mathrm{a}, 1 \mathrm{c}, 1 \mathrm{~d}, 1 \mathrm{~g}$ \\
\hline 1c. $20-24$ & 38.923 & 33.616 & 1,158 & $\begin{array}{c}1 \mathrm{a}, 1 \mathrm{~b}, 1 \mathrm{c}, 1 \mathrm{~d} \\
1 \mathrm{f}, 1 \mathrm{~g}\end{array}$ \\
\hline 1d. $25-29$ & 31.098 & 26.503 & 1,173 & $1 \mathrm{a}, 1 \mathrm{~b}, 1 \mathrm{e}, 1 \mathrm{f}, 1 \mathrm{~g}$ \\
\hline 1e. $30-34$ & 23.011 & 19.242 & 1,196 & $1 \mathrm{a}, 1 \mathrm{c}, 1 \mathrm{~d}, 1 \mathrm{~g}$ \\
\hline 1f. $35-39$ & 15.589 & 13.001 & 1,199 & $1 \mathrm{a}, 1 \mathrm{c}, 1 \mathrm{~d}, 1 \mathrm{~g}$ \\
\hline 1g. 40 e mais & 7.261 & 5.917 & 1,227 & $1 a, 1 c, 1 d$ \\
\hline \multicolumn{5}{|c|}{ 2. Escolaridade da gestante (anos) } \\
\hline 2a. Nenhuma & 12.525 & 10.165 & 1,232 & $2 b, 2 c, 2 d, 2 e$ \\
\hline 2b. $1-3$ & 20.460 & 17.501 & 1,169 & $2 a$ \\
\hline 2c. $4-7$ & 40.310 & 33.889 & 1,189 & $2 a, 2 b$ \\
\hline 2d. $8-11$ & 31.614 & 26.671 & 1,185 & $2 a, 2 b$ \\
\hline 2e. 12 e mais & 11.257 & 9.528 & 1,181 & $2 a$ \\
\hline \multicolumn{5}{|c|}{ 3. Duração da gestação (semanas) } \\
\hline 3a. $<22$ & 8.828 & 5.968 & 1,479 & $3 b, 3 c, 3 d, 3 e, 3 f$ \\
\hline 3b. $22-27$ & 38.837 & 32.585 & 1,192 & $3 a, 3 c, 3 d, 3 f$ \\
\hline 3c. $28-31$ & 27.487 & 24.230 & 1,134 & $3 a, 3 b, 3 d, 3 e, 3 f$ \\
\hline $3 d .32-36$ & 42.841 & 36.734 & 1,166 & $3 a, 3 b, 3 c, 3 e$ \\
\hline 3e. $37-41$ & 42.378 & 35.699 & 1,187 & $3 a, 3 c, 3 d$ \\
\hline 3f. $\geq 42$ & 3.138 & 2.684 & 1,169 & $3 a$ \\
\hline
\end{tabular}

além de um sentimento pessoal de vergonha referente à exposição pública da sua sexualidade precoce 17 . O efeito da ausência de escolaridade por parte da gestante, por outro lado, está associado à menor renda, menor acesso a serviços de saúde e maior vulnerabilidade social 18. A gestação de curta duração (menos de 22 semanas) pode ser tanto uma consequência dessas situações de risco apresentadas pela gestante, como também um desencadeador de maior vulnerabilidade ${ }^{19}$. Além das características específicas das gestantes, estima-se que a realidade brasileira de maior risco social (nos quesitos renda e violência) possa aumentar os níveis gerais de estresse e levar a uma maior RM fetal em relação a países mais desenvolvidos e estáveis 20,21.

É importante mencionar que a presença de fatores de risco e condições externas de estresse não são os únicos prováveis determinantes da maior mortalidade fetal masculina. Estudos feitos por Christiansen et al. ${ }^{22}$, por exemplo, apontam para a importância das respostas imunológicas maternas ao antígeno de histocompatibilidade
H-Y no aumento do número de perdas fetais subsequentes. Um estudo de Nielsen 23 verificou, ao testar a teoria, que essas perdas fetais são maiores para o sexo masculino.

As variáveis analisadas pelo presente estudo podem também ter efeito relativo na diferença entre as razões de masculinidade, independentemente das condições sociais e biológicas específicas da gestante. Um estudo de 3.904 necrópsias, realizado em Curitiba (Paraná), verificou que, conforme o período gestacional aumenta, as causas básicas de óbitos fetais se assemelham às verificadas no período neonatal 24. Portanto, existe uma tendência natural de as razões de masculinidade dos óbitos fetais ocorridos após 22 semanas de gestação se aproximarem das observadas para os recém-nascidos.

Pesquisas brasileiras que analisaram a mortalidade fetal e perinatal (sem distinção por sexo) encontraram maior mortalidade entre algumas das variáveis analisadas por este estudo, como para baixa escolaridade 9,11 , mas não para idade da gestante ${ }^{10}$. Uma investigação realizada no Rio 
Causas básicas mais frequentes entre óbitos fetais segundo sexo e presença de diferença estatisticamente significativa em relação à razão de masculinidade (RM) de 1,05. Brasil, 2000 a 2009.

\begin{tabular}{|c|c|c|c|c|c|c|c|c|c|c|c|c|c|}
\hline \multirow[t]{2}{*}{ CID-10 } & \multirow[t]{2}{*}{ Causa básica } & \multicolumn{3}{|c|}{ Total } & \multicolumn{3}{|c|}{10 a 14 anos } & \multicolumn{3}{|c|}{ Nenhuma escolaridade } & \multicolumn{3}{|c|}{ Menos de 22 semanas } \\
\hline & & Masculino & Feminino & RM & Masculino & Feminino & $\mathrm{RM}$ & Masculino & Feminino & RM & Masculino & Feminino & $\mathrm{RM}$ \\
\hline A50 & Sífilis congênita & 396 & 371 & 1,07 & 10 & 4 & 2,50 & 21 & 24 & 0,88 & 15 & 12 & 1,25 \\
\hline $\mathrm{POO}$ & Afecções maternas & 18.155 & 15.885 & 1,14 * & 101 & 99 & 1,02 & 1.079 & 930 & 1,16 * & 337 & 275 & 1,23 * \\
\hline P01 & $\begin{array}{l}\text { Complicações } \\
\text { maternas da gravidez }\end{array}$ & 6.457 & 5.117 & 1,26 * & 80 & 55 & 1,45 * & 377 & 291 & 1,30 * & 721 & 448 & 1,61 * \\
\hline P02 & $\begin{array}{l}\text { Complicações da } \\
\text { placenta, cordão } \\
\text { umbilical e membrana }\end{array}$ & 37.852 & 30.936 & 1,22 * & 367 & 241 & 1,52 * & 2.746 & 2.168 & 1,27 * & 1.257 & 825 & 1,52 * \\
\hline P03 & $\begin{array}{l}\text { Complicações do } \\
\text { trabalho de parto e } \\
\text { parto }\end{array}$ & 2.398 & 1.845 & 1,30 * & 36 & 29 & 1,24 & 231 & 153 & 1,51 * & 131 & 94 & 1,39 * \\
\hline P04 & $\begin{array}{l}\text { Inflamações nocivas } \\
\text { transmitidas via } \\
\text { placenta }\end{array}$ & 304 & 273 & 1,11 & 3 & 2 & 1,50 & 29 & 17 & 1,71 & 20 & 9 & 2,22 * \\
\hline P05 & $\begin{array}{l}\text { Crescimento } \\
\text { fetal retardado e } \\
\text { desnutrição }\end{array}$ & 921 & 735 & 1,25 * & 11 & 16 & 0,69 & 69 & 50 & 1,38 & 86 & 59 & 1,46 * \\
\hline P07 & $\begin{array}{l}\text { Gestação de curta } \\
\text { duração e baixo peso }\end{array}$ & 1.036 & 689 & 1,50 * & 23 & 15 & 1,53 & 50 & 42 & 1,19 & 162 & 110 & 1,47 * \\
\hline P20 & Hipóxia intrauterina & 47.016 & 39.752 & 1,18 * & 462 & 362 & 1,28 * & 2.822 & 2.288 & 1,23 * & 2.271 & 1.569 & 1,45 * \\
\hline P35-39 & $\begin{array}{l}\text { Infecções específicas } \\
\text { do período perinatal }\end{array}$ & 609 & 499 & 1,22 * & 9 & 8 & 1,13 & 57 & 36 & 1,58 * & 26 & 11 & 2,36 * \\
\hline P55 & $\begin{array}{l}\text { Doença hemolítica } \\
\text { do feto }\end{array}$ & 404 & 328 & 1,23 * & 2 & 0 & - & 34 & 15 & 2,27 * & 7 & 6 & 1,17 \\
\hline P70 & $\begin{array}{l}\text { Transtorno transitório } \\
\text { do metabolismo dos } \\
\text { carboidratos }\end{array}$ & 1.092 & 794 & 1,38 * & 0 & 0 & - & 70 & 58 & 1,21 & 3 & 3 & 1,00 \\
\hline P83 & $\begin{array}{l}\text { Afecções que } \\
\text { comprometem o } \\
\text { tegumento }\end{array}$ & 511 & 540 & $0.95^{\star \star}$ & 3 & 6 & 0,50 & 23 & 29 & 0,79 & 22 & 36 & $0,61 * *$ \\
\hline P95 & $\begin{array}{l}\text { Morte de causa fetal } \\
\text { não especificada }\end{array}$ & 52.904 & 43.994 & 1,20 * & 610 & 408 & 1,50 * & 3.918 & 3.181 & 1,23 * & 3.108 & 1.998 & 1,56 * \\
\hline P96 & $\begin{array}{l}\text { Outras afecções } \\
\text { originadas no período } \\
\text { perinatal }\end{array}$ & 6.024 & 4.938 & 1,22 * & 73 & 69 & 1,06 & 467 & 364 & 1,28 * & 451 & 307 & 1,47 * \\
\hline Q00 & Anencefalia & 1.431 & 2.420 & 0,59 ** & 18 & 29 & 0,62 ** & 88 & 128 & $0,69 * *$ & 24 & 32 & 0,75 \\
\hline Q03 & Hidrocefalia congênita & 459 & 342 & 1,34 * & 2 & 3 & 0,67 & 37 & 20 & 1,85 * & 6 & 5 & 1,20 \\
\hline Q20-28 & $\begin{array}{l}\text { Malformações } \\
\text { congênitas do } \\
\text { aparelho circulatório }\end{array}$ & 662 & 611 & 1,08 & 0 & 1 & 0,00 & 28 & 36 & 0,78 & 7 & 6 & 1,17 \\
\hline Q89 & $\begin{array}{l}\text { Outras malformações } \\
\text { congênitas NCOP }\end{array}$ & 3.218 & 2.964 & 1,09 & 29 & 27 & 1,07 & 204 & 191 & 1,07 & 98 & 96 & 1,02 \\
\hline
\end{tabular}

* $p<0,05$

** Nesses casos, a RM é estatisticamente menor que 1,05 ( $p<0,05)$. 
de Janeiro encontrou associação entre sexo masculino e maior mortalidade neonatal 10.

Outra variável de interesse para óbitos fetais é a análise da RM segundo raça/cor. Estudos realizados nos Estados Unidos mostram que, nos últimos anos, tem sido verificada uma diminuição da RM de recém-nascidos entre brancos, mas não entre negros 25 . Entretanto, essa análise, apesar de disponível pelo Sistema de Informações sobre Nascidos Vivos (SINASC), não foi possível para o presente estudo em virtude do extremo número de óbitos com cor/raça ignorados no período $(98,6 \%)$.

Entre as variáveis analisadas, o total de ignorados foi de $20,3 \%$ para idade da gestante, $37,1 \%$ para escolaridade da gestante e $11,3 \%$ para duração da gestação, o que pode ser um fator limitante para o presente estudo. Resultados equivalentes foram encontrados em uma pesquisa sobre óbitos fetais em São Paulo 26. Além da presença de variáveis não informadas, outra limitação é o problema da subnotificação de óbitos. Um trabalho realizado em Pernambuco, que comparou os dados oficiais do SIM com os de uma investigação realizada em cartórios, unidades de saúde, cemitérios e comunidades, encontrou uma subenumeração de $45,8 \%$ dos óbitos infantis locais 27.

O desequilíbrio na distribuição dos sexos é uma preocupação crescente para vários países. Em alguns, verifica-se excesso de homens, co- mo no caso da China e da Índia, onde o aborto seletivo de fetos femininos tem aumentado por causa da disseminação do uso de aparelhos de ultrassom 28 . Por outro lado, países com alto nível de violência urbana e guerras têm apresentado um excesso de mulheres em idade fértil 29 . No caso recente da Rússia, essa desproporção tem ocorrido pelo aumento da mortalidade masculina por causas básicas relacionadas ao abuso de substâncias alcoólicas 30 .

A diferença na distribuição de sexo na população pode levar a um grande número de problemas sociais. Entre estes, destacam-se revoltas e tráficos de seres humanos, como no caso recente da China, onde se estima que, atualmente, 40 milhões de homens não consigam encontrar esposas 31 .

Apesar do crescimento da importância da mortalidade materna e infantil, em virtude da sua inclusão nos objetivos do desenvolvimento do milênio da Organização das Nações Unidas (ONU), a análise da mortalidade fetal tem sido deixada de lado, sendo excluída dos estudos de carga global de doenças 32 . Pesquisas recentes estimam que cerca de 2,65 milhões de óbitos fetais ocorram todos os anos, sendo $98 \%$ destes em países em desenvolvimento ${ }^{33}$. Logo, entender os padrões de vulnerabilidade para óbitos fetais torna-se importante, seja para prevenir a mortalidade pré-natal, seja para evitar um desequilíbrio na distribuição dos sexos.

\section{Resumo}

Alguns estudos apontam para a existência de vulnerabilidades biológicas inatas masculinas, especialmente no período perinatal. Foi realizada uma análise transversal da mortalidade fetal brasileira segundo sexo, entre 2000 e 2009 (inclusive), conforme características maternas (idade, escolaridade e duração da gestação), utilizando-se dados disponibilizados pelos sistema DATASUS do Ministério da Saúde. Todos os óbitos fetais do período foram incluídos na análise, excetuando-se os casos em que o sexo do feto não foi declarado. A razão de masculinidade (RM) encontrada para os óbitos fetais foi de 1,188. As categorias mais relacionadas com maior risco (idade entre 10 e 14 anos, nenhuma escolaridade e gestação com menos de 22 semanas) apresentaram maior RM, sendo esses valores, em todos os casos, estatisticamente maiores do que os observados nas outras categorias analisadas $(p<0,05)$. Verificou-se RM estatisticamente maior $(p<0,05)$ ao esperado para 13 causas básicas de óbito e menor para duas. Os resultados encontrados pelo estudo apontam para uma possível vulnerabilidade biológica inata masculina.

Razão de Masculinidade; Mortalidade Fetal; Saúde do Homem 


\section{Colaboradores}

A. D. P. Chiavegatto Filho participou da elaboração da pesquisa, análise dos resultados, interpretação dos resultados, redação do artigo e aprovação do texto final. R. Laurenti contribuiu na elaboração da pesquisa, análise e interpretação dos resultados, revisão crítica do conteúdo do artigo e aprovação do texto final.

\section{Referências}

1. Laurenti R, Mello-Jorge MHP, Gotlieb SLD. Perfil epidemiológico da morbi-mortalidade masculina. Ciênc Saúde Coletiva 2005; 10:35-46.

2. Bird CE, Rieker PP. Gender matters: an integrated model for understanding men's and women's health. Soc Sci Med 1999; 48:745-55.

3. Pinheiro RS, Viacava F, Travassos C, Brito AS. Gênero, morbidade, acesso e utilização de serviços de saúde no Brasil. Ciênc Saúde Coletiva 2002; 7 : 687-707.

4. Drevenstedt GL, Crimmins EM, Vasunilashorn S, Finch CE. The rise and fall of excess male infant mortality. Proc Natl Acad Sci U S A 2008; 105: 5016-21.

5. Gupta MD. Explaining Asia's "missing women": a new look at the data. Popul Dev Rev 2005; 31: 529-35.

6. Bruckner TA, Catalano R, Ahern J. Male fetal loss in the U.S. following the terrorist attacks of September 11, 2001. BMC Public Health 2010; 10:273.

7. Obel C, Henriksen TB, Secher NJ, Eskenazi B, Hedegaard M. Psychological distress during early gestation and offspring sex ratio. Hum Reprod 2007; 22:3009-12.

8. MacDorman MF, Kirmeyer S. Fetal and perinatal mortality, United States, 2005. Natl Vital Stat Rep 2009; 57:1-19.

9. Fonseca SC, Coutinho ESF. Pesquisa sobre mortalidade perinatal no Brasil: revisão da metodologia e dos resultados. Cad Saúde Pública 2004; 20 Suppl 1:S7-19.

10. Oliveira EFV, Gama SGN, Silva CMFP. Gravidez na adolescência e outros fatores de risco para mortalidade fetal e infantil no Município do Rio de Janeiro, Brasil. Cad Saúde Pública 2010; 26:567-78.

11. Almeida MF, Alencar GP, Novaes HMD, França Jr. I, Siqueira AAF, Campbell OMR, et al. Risk-factors for antepartum fetal deaths in the city of São Paulo, Brazil. Rev Sáude Pública 2007; 41:35-43.

12. Menezes AMB, Barros FC, Victora CG, Tomasi E, Halpern R, Oliveira ALB. Fatores de risco para mortalidade perinatal em Pelotas, RS, 1993. Rev Saúde Pública 1998; 32:209-16.

13. Lansky S, França E, Leal MC. Mortalidade perinatal e evitabilidade: revisão da literatura. Rev Saúde Pública 2002; 36:259-72.

14. Conselho Federal de Medicina, Ministério da Saúde. A declaração de óbito: documento necessário e importante. 2a Rev. Brasília: Centro Brasileiro de Classificação de Doenças; 2007.

15. Organização Mundial da Saúde. Classificação estatística internacional de doenças e problemas relacionados à saúde. 10a Rev. São Paulo: Edusp; 2003. 
16. United Nations. Live births by age of mother, sex of the child and urban/rural residence: latest available year, 1995-2004. Table 10. http://unstats.un.org/ unsd/demographic/products/dyb/DYB2004/Ta ble10.pdf (acessado em 20/Ago/2011).

17. Dadoorian D. Gravidez na adolescência: um novo olhar. Psicol Ciênc Prof 2003; 23:84-91.

18. Haidar FH, Oliveira UF, Nascimento LFC. Escolaridade materna: correlação com os indicadores obstétricos. Cad Saúde Pública 2001; 17:1025-9.

19. Zeitlin J, Saurel-Cubizolles MJ, Mouzon J, Rivera L, Ancel PY, Blondel B, et al. Fetal sex and preterm birth: are males at greater risk? Hum Reprod 2002; 17:2762-8.

20. Khashan AS, Mortensen PB, McNamee R, Baker $\mathrm{PN}$, Abel KM. Sex ratio at birth following prenatal maternal exposure to severe life events: a population-based cohort study. Hum Reprod 2009; 24:1754-7.

21. Navara KJ. Programming of offspring sex ratios by maternal stress in humans: assessment of physiological mechanisms using a comparative approach. J Comp Physiol B 2010; 180:785-96.

22. Christiansen OB, Pedersen B, Nielsen HS, Nybo Andersen A-M. Impact of the sex of first child on the prognosis in secondary recurrent miscarriage. Hum Reprod 2004; 19:2946-51.

23. Nielsen HS. Secondary recurrent miscarriage and H-Y immunity. Hum Reprod Update 2011; 17: 558-74

24. Noronha L, Martins VDM, Nones RB, Sepulcri RP, Carvalho DS, Sampaio GA, et al. Mortalidade intrauterina e perinatal: análise comparativa de 3.904 necrópsias do Hospital de Clínicas de Curitiba no período de 1960 a 1995. J Pediatr (Rio J.) 2000; 76:213-21.
25. Davis DL, Webster P, Stainthorpe H, Chilton J, Jones L, Doi R. Declines in sex ratio at birth and fetal deaths in Japan, and in U.S. whites but not African Americans. Environ Health Perspect 2007; 115:941-6.

26. Beringhs EM, Gallo PR, Reis AOA. Declarações de nascidos mortos no município de São Paulo: avaliação descritiva do preenchimento. Rev Bras Saúde Matern Infant 2008; 8:319-23.

27. Frias PG, Vidal SA, Pereira PMH, Lira PIC, Vanderlei LC. Avaliação da notificação de óbitos infantis ao Sistema de Informações sobre Mortalidade: um estudo de caso. Rev Bras Saúde Matern Infant 2005; 5 Suppl 1:S43-52.

28. Sen A. Missing women - revisited. BMJ 2003; 327:1297.

29. Barber N. Marriage markets and mating aggression help explain societal differences in violent crime. Agress Violent Behav 2011; 16:420-7.

30. Vallin J, Meslé F, Valkonen T. Trends in mortality and differential mortality. Strasburg: Council of Europe Publishing; 2001.

31. McCurry J, Allison R. 40M bachelors and no women... the birth of a new problem for China. The Guardian 2004; March 9. http://www.guardian. co.uk/world/2004/mar/09/china.justinmccurry (acessado em 20/Jul/2011).

32. Frøen JF, Cacciatore J, McClure EM, Kuti O, Jokhio AH, Islam M, et al. Stillbirths: why they matter. Lancet 2011; 377:1353-66.

33. Lawn JE, Blencowe H, Pattinson R, Cousens S, Kumar R, Ibiebele I, et al. Stillbirths: Where? When? Why? How to make the data count? Lancet 2011; 377:1448-63.

Recebido em 24/Ago/2011

Versão final reapresentada em 09/Nov/2011

Aprovada em 13/Dez/2011 